\title{
Construcciones de adobe resistentes a exposición prolongada de agua por efecto de inundaciones
}

\section{Resistant adobe constructions under prolonged water exposure caused by floods}

\author{
*Miguel Ángel Trujillo Barrera ${ }^{1}$, Jorge Chavez Guivin², Elías Alberto Torres Armas ${ }^{3}$
}

\section{RESUMEN}

La presente tesis de investigación tuvo como objetivo principal evaluar una construcción de adobe resistente a una exposición prolongada de agua por inundación. Se implementaron tres alternativas: muro con sobrecimiento de concreto simple, muro con sobrecimiento de ladrillo, un muro con sobrecimiento tarrajeado; y un muro sin protección (testigo). Se realizó ensayos de succión y absorción de unidades, además de prueba de inundación simulada; y se determinó el desempeño de las estructuras ante periodos cortos y prolongados de exposición al agua. Se encontró que los muros con sobrecimiento de concreto simple presentan mayor resistencia a inundaciones frente a muros con sobrecimiento de ladrillo y muros con sobrecimiento tarrajeado.

Palabras clave: adobe, succión, absorción, inundación simulada.

\begin{abstract}
The main purpose of this work is to evaluate and to determine a resistant adobe construction under prolonged water exposure caused by floods. The three alternatives were implemented: a wall with simple concrete overlay, a wall with brick overlay or a wall with tarred overlay; and a wall without protection (witness). Suction and unit absorption tests were carried out, as well as simulated flood test; and the structures response to the prolonged periods of water exposure was determined. It was found that the walls with simple concrete overlay have greater resistance to flooding in comparison with other types of walls.
\end{abstract}

Keywords: adobe, suction, absorption, flood simulation.

\footnotetext{
${ }^{1}$ *Ingeniero Civil. Universidad Nacional Toribio Rodríguez de Mendoza de Amazonas. Correo electrónico: miguelangel t b@hotmail.com

${ }^{2}$ Ingeniero Civil. Universidad Nacional Toribio Rodríguez de Mendoza de Amazonas

${ }^{3}$ Licenciado en Estadística. Universidad Nacional Toribio Rodríguez de Mendoza de Amazonas
} 


\section{INTRODUCCIÓN}

El barro ha sido desde los inicios de la humanidad, el material de construcción más utilizado y una de sus formas de utilización es el adobe, con los que se construyen paredes y tabiques de muy buena estabilidad (Canchig y Del Rocío (2007).

En el Perú, el uso del adobe es muy común en zonas rurales, donde la autoconstrucción con adobe ha demostrado ser la respuesta apropiada y quizás, la única vía posible mediante la cual la gente de muy escasos recursos económicos pueda adquirir una casa digna. El adobe es un material muy barato y que tiene un gran aislamiento térmico haciendo estas casas muy acogedoras. El gran problema surge cuando en las construcciones de estas viviendas no se cuenta con asesoría técnica y se construyen de manera muy informal, llevando esta mala construcción al colapso ante alguna eventualidad. Las casas de adobe bien diseñadas y bien construidas pueden ser, por su simplicidad, duración y costo, la base para resolver el problema de la vivienda, sobretodo la del tipo rural (Delgado, 2006).

Al igual que las acciones sísmicas, el enemigo de las edificaciones de adobe es la humedad, principalmente cuando quedan expuestas prolongadamente a inundaciones. El agua desintegra la unión que existe entre las partículas que conforman al adobe, convirtiéndolo en barro, lo que produce el colapso de las edificaciones. (Cabrera \& Huaynate, 2010).

Se han realizado muchos trabajos que evalúan las construcciones de adobe y han permitido verificar su versatilidad, y con la incorporación de ciertos complementos pueden significar una muy buena alternativa de construcción (Ottazzi, Felipe, Blondet, Villa-García y Ginocchio, 1989; Ginocchio, Quiun y Cadillo, 2014).

Por lo que el objetivo de esta investigación fue evaluar y determinar una construcción de adobe resistente a una exposición prolongada de agua.

\section{MATERIAL Y MÉTODOS}

\section{Materiales utilizados}

\section{Adobe}

Para el ensayo se elaboraron adobes, de acuerdo al acápite 4.2 Formas y Dimensiones, de la Norma E. 080 , de $20 \times 33 \times 13 \mathrm{~cm}$.

\section{Mortero}

Se utilizó mortero con cemento Portland tipo I y arena de cerro gruesa tamizada libre de impurezas, con una dosificación en volumen cemento-arena 1:4.

\section{Mezcla para tarrajeo de muros}

Se utilizó una mezcla uniforme de arena fina y cemento Portland tipo I. La dosificación volumétrica cemento-arena utilizada fue 1:4.

\section{Concreto simple para sobrecimiento}

El concreto utilizado tuvo una resistencia a la compresión $\mathrm{f}^{\prime} \mathrm{c}=210 \mathrm{~kg} / \mathrm{cm}^{2}$, con una dosificación en peso de 1:2,45:2,30 $+20,81 \mathrm{~L} /$ bolsa de cemento y una dosificación en volumen de 1:2,06:2,25 + 20,81 L/bolsa de cemento.

\section{Diseño del experimento}

Se evaluó tres alternativas y un muro patrón (MP): T1: muro con sobrecimiento de concreto simple previo a la construcción del adobe (MC); T2: empleo de ladrillos de arcilla industrial tipo IV King Kong de 18 huecos, en la base de los muros de adobe (ML) y T3: la protección de la base de los muros de adobe con tarrajeo de cemento (MT).

\section{Ensayo de inundación simulada en los muros}

Se utilizó un canal de concreto armado con sección transversal en forma de $\mathrm{U}$ de $40 \mathrm{~cm}$ de ancho interior. Se dividió en sus 4 lados colocando barreras en las esquinas que impidan el paso del agua entre sectores y se impermeabilizará cada sector por separado. Luego, se edificó cuatro muros por separado (tratamientos) y se dejó secar a humedad ambiente durante 3 semanas.

Para medir la cantidad de agua absorbida por cada muro, se colocó una mira a manera de cartilla de medición inversa en cada sector del canal, graduado al centímetro y con una altura de $30 \mathrm{~cm}$.

La cantidad de agua que sube a través de cada muro por capilaridad, se midió con niveles topográficos, instalados desde 30 a $60 \mathrm{~cm}$ de la base, con separaciones cada $5 \mathrm{~cm}$ y con colores fáciles de identificar y medir.

Posteriormente, se inundó los cuatro sectores con un volumen conocido de agua, procurando que el llenado de agua sea lo más rápido y constante posible.

Se evaluó dos periodos: Periodo Corto de Inundación (PCI), durante 72 horas y Periodo Prolongado de Inundación (PPI), desde el final del PCI hasta dos semanas.

Los tratamientos que no soportaron el PCI, fueron catalogados como alternativas que no resisten a la inundación; los muros que colapsaron durante el PPI, fueron catalogados como resistentes a la inundación, pero sufre daños irreparables y los muros que se 
mantuvieron en pie fueron considerados exitosos y soportan la inundación.

Durante el PCI, se realizaron mediciones de absorción y capilaridad a cada hora y cada 24 horas se repuso el volumen de agua perdida por absorción.

En el PPI se realizó mediciones de absorción y capilaridad cada 24 horas y al cabo de cada semana se repuso el volumen de agua perdida por absorción, para no detener el ascenso por capilaridad. Durante este periodo se dilatan los tiempos de medición y reposición de agua por lo que no es necesario una grabación continua durante el transcurso de las 2 semanas, por lo que, sólo se documentó mediante fotografías diarias.

Con la información recogida se obtuvo: el tiempo de resistencia a la inundación, tiempo vs altura de capilaridad y tiempo vs altura de agua absorbida.

\section{Prueba de succión en unidades}

La prueba de periodo inicial de succión, se basó en la técnica NTP 399.613 acápite 11, que tiene por objetivo obtener el índice de succión temprana del espécimen ensayado; la que consiste en determinar la cantidad de agua que la unidad de adobe puede absorber en un periodo normado de $1 \mathrm{~min} \pm 1 \mathrm{~s}$.

\section{Prueba de absorción en unidades}

El ensayo se realizó conforme lo estipula la NTP 399.613 acápite 11 , para conocer la capacidad de absorción del adobe durante 24 horas de inmersión en agua a una temperatura de $24^{\circ} \mathrm{C} \pm 8^{\circ} \mathrm{C}$.

\section{Determinación de la integridad}

Para cuantificar la integridad de los muros, se realizó una comparación mediante humedad y estabilidad durante el ensayo; peso por humedad mediante la inspección visual y táctil realizada en las unidades de adobe al momento de la demolición, y la solidez de las bases inundadas al terminar el ensayo.

\section{Determinación del índice de daño (ID)}

Se cuantificó de acuerdo a proporciones al mejor de los métodos, en la proporción cero (0.00) (si es que no sufre daño alguno) y los demás métodos se les asignaron un peso respecto al mejor. Luego se colocó dichas proporciones en una matriz comparativa, se les asignó porcentajes de incidencia a cada aspecto y del producto de daño comparativo por el peso asignado se obtuvo el ID ante inundaciones. Mientras más bajo sea el ID, mejor es la alternativa de mejora.

Las variables capilaridad, absorción, humedad, estabilidad y solidez de la base de los muros, fueron integradas siguiendo el procedimiento descrito por
Cabrera y Huaynate (2010) y Romero y Pereyra (2012).

\section{RESULTADOS}

El muro con sobrecimiento de concreto simple, fue el que obtuvo el menor índice de daño a inundaciones, seguido por el muro con sobrecimiento de ladrillo y el muro tarrajeado, tal como se observa en la Tabla 1 y Figura 1.

El muro tarrajeado evidencia una nula absorción de agua, sin embargo tuvo la mayor inconsistencia en la base y mayor humedad.

Tabla 1. Matriz de cuantificación de daños ante inundaciones

\begin{tabular}{|c|c|c|c|c|c|}
\hline 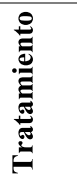 & 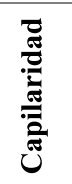 & 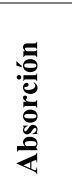 & 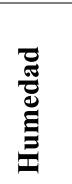 & 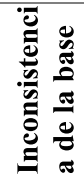 & 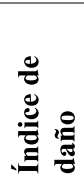 \\
\hline Peso & 10 & 30 & 10 & 50 & 100 \\
\hline $\begin{array}{l}\text { T1 } \\
\text { (MC) }\end{array}$ & 0,21 & 0,17 & 0,00 & 0,00 & 7,20 \\
\hline $\begin{array}{l}\text { T2 } \\
\text { (ML) }\end{array}$ & 0,00 & 1,15 & 0,00 & 0,00 & 34,50 \\
\hline $\begin{array}{l}\text { T3 } \\
\text { (MT) }\end{array}$ & 0,06 & 0,00 & 0,45 & 1,00 & 55,10 \\
\hline
\end{tabular}

En cuanto al costo, los muros con sobrecimiento de concreto simple, son los más caros y el muro patrón el más barato (Figura 2).

La Tabla 2, muestra los valores integrados de las tres alternativas y el testigo evaluado, donde se evidencia que todas las alternativas superaron a la técnica tradicional, donde las alternativas más viables son el uso de sobrecimiento de concreto simple y de ladrillo.

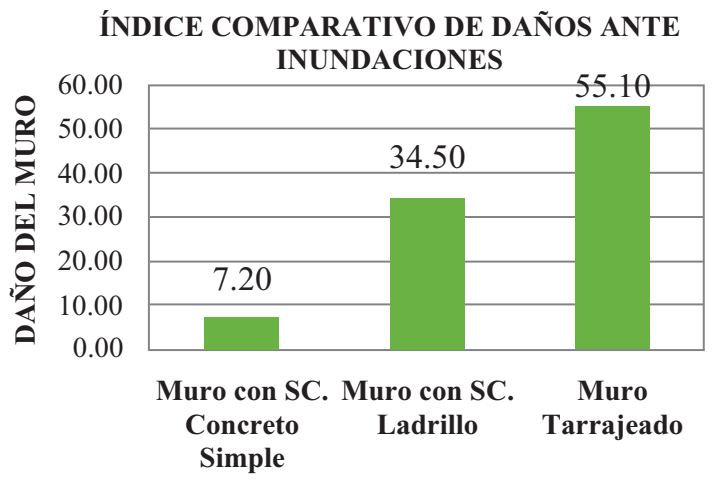

TIPO DE MUROS

Figura 1. Daños estructurales sufridos de los tres muros 


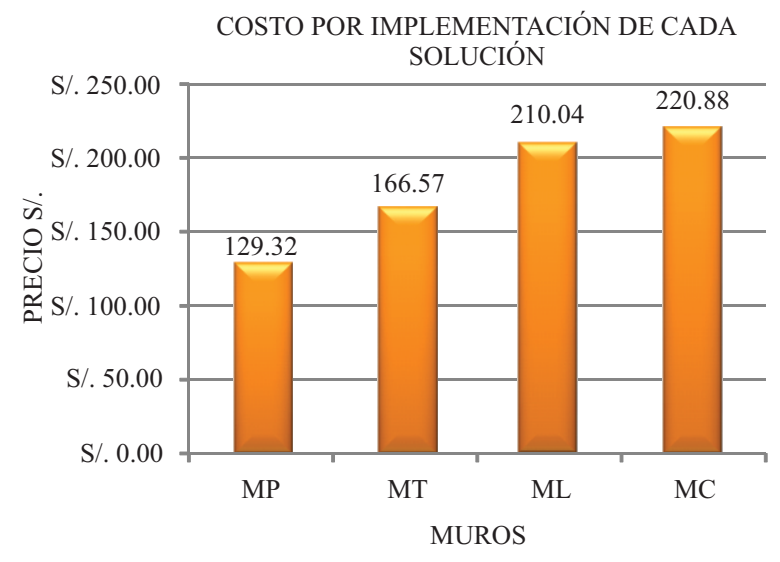

Figura 2. Costo por construcción de cada solución propuesta

Tabla 2. Porcentaje de daño comparativo por costo

\begin{tabular}{|c|c|c|c|c|}
\hline & 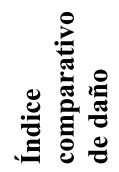 & 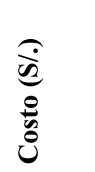 & 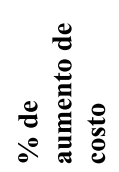 & 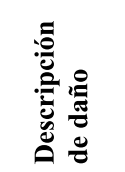 \\
\hline $\begin{array}{l}\text { Muro } \\
\text { Patrón }\end{array}$ & Colapso & 129,32 & 0,00 & Colapso \\
\hline $\begin{array}{l}\text { T1 (Muro } \\
\text { con SC de } \\
\text { concreto } \\
\text { simple) }\end{array}$ & 7,20 & 220,88 & 70,79 & $\begin{array}{l}\text { Sin daños } \\
\text { graves }\end{array}$ \\
\hline $\begin{array}{l}\text { T2 } \\
\text { (Muro con } \\
\text { SC de } \\
\text { ladrillo) }\end{array}$ & 34,50 & 210,04 & 62,42 & $\begin{array}{l}\text { Sin daños } \\
\text { graves }\end{array}$ \\
\hline $\begin{array}{l}\text { T3 (Muro } \\
\text { tarrajeado) }\end{array}$ & 55,10 & 166,57 & 28.69 & $\begin{array}{l}\text { Daño } \\
\text { grave }\end{array}$ \\
\hline
\end{tabular}

\section{DISCUSIÓN}

Concordando con Cabrea y Huaynate (2010), se evidencia que las construcciones con sobrecimiento de concreto simple presentan mejores resultados frente a inundaciones, incluso con un sobrecimiento de hasta $5 \mathrm{~cm}$ menor al evaluado por los autores precitados.

Se verificó que las construcciones de adobe con sobrecimiento de concreto simple son mejores que muros con sobrecimiento de ladrillo, contrastando lo reportado por Romero y Pereira (2012), quienes mencionan que el ladrillo presenta mejores comportamientos frente a inundaciones que el concreto ciclópeo para sobrecimiento.

Las construcciones de adobe, con ciertas mejoras tecnológicas, pueden ser empleadas en la construcción de viviendas, en zonas susceptibles de sufrir inundaciones, tal como evidenciara Ginocchio, Quiun y Cadillo (2014).

\section{CONCLUSIONES}

El método de construcción a base de tierra que se propone y que presentó mejores resultados ante el colapso por inundación prolongada, fue la utilización de un sobrecimiento de concreto simple en la base de muro de adobe convencional, con un peralte de $25 \mathrm{~cm}$ mayor que la altura de agua esperada.

Aumentando el costo del muro patrón en 70,79\% y $62,42 \%$, mediante una solución con sobrecimiento de concreto simple o con sobrecimiento de ladrillo respectivamente, el daño por inundación es nulo, mientras que aumentando el costo del muro patrón en $28,7 \%$, mediante las soluciones de adobe con tarrajeo de la base, se logra evitar el colapso del adobe convencional ante las inundaciones.

\section{REFERENCIAS BIBLIOGRÁFICAS}

Cabrera, A., y Huaynate, G. (2010). Mejoramiento de las construcciones de adobe ante una exposición prolongada de agua por efecto de inundaciones (Tesis para optar el título de ingeniero civil). Pontificia Universidad Católica del Perú. Lima, Perú.

Canchig, J., y del Rocío, S. (2007). Uso del adobe como material de construcción (Tesis de grado). Escuela Politécnica Nacional, Quito-Ecuador.

Delgado, C. (2006). Comportamiento sísmico de un módulo de adobe de dos pisos con refuerzo horizontal y confinamientos de concreto armado (Tesis para optar el título de ingeniero civil). Pontificia Universidad Católica del Perú, Lima, Perú.

Ginocchio, J. F., Quiun, D. R., y Cadillo, A. D. (2014). Inspección de estructuras de adobe construidas en programas de vivienda desarrollados hace más de veinticinco años en el norte de Perú. Disponible en https://www.researchgate.net/profile/Danie 1_Quiun/publication/304579274

Hernández, R. (2006). Metodología de la investigación ( $4^{\mathrm{a}}$ ed.). Editorial. McGrawHill.

INDECOPI. (2005). Unidades de Albañilería. Métodos de muestreo y ensayos en ladrillos de arcilla usados en albañilería. Norma Técnica Peruana NTP 399.6132005. Comisión de Reglamentos Técnicos y comerciales. Lima, Perú.

Ottazzi, G., Yep, J., Blondet, M., Villa-Garcia, G., y 
Ginocchio, J. (1989). Ensayos de simulación sísmica de viviendas de adobe. Seismic Simulation Tests of Adobe Houses). Research Project Financed by the International Development Research Centre (IDRC-Canada). Publication DI-89-01, Departamento de Ingeniería, Pontificia Universidad Católica del Perú, Lima, Perú.

Romero, B., y Pereyra, M. (2012). Mejoramiento de las construcciones de adobe ante una exposición prolongada de agua por efecto de inundaciones - parte 2 (Tesis para optar el título de ingeniero civil). Pontificia Universidad Católica del Perú. Lima, Perú. 